This is the accepted manuscript of the article, which has been published in Scandinavian Journal of Caring Sciences. https://doi.org/10.1111/scs.12770

Paula Yliluoma (corresponding author at)

MNSc, Public Health Nurse

University of Tampere, Faculty of Social Sciences

paula.yliluoma@gmail.com

\title{
Mira Palonen
}

$\mathrm{PhD}, \mathrm{RN}$, University Teacher

University of Tampere, Faculty of Social Sciences

mira.palonen@staff.uta.fi 


\section{Abstract}

Background: Telephone nursing is expanding worldwide, but little is known about nurses' interactions with callers and the factors that affect these interactions.

Aim: The purpose of this paper is to describe how telenurses experience caller interactions.

Methods: A qualitative study designed through open telephone interviews with call centre nurses $(\mathrm{n}=9)$ in 2017. The data was analysed using inductive content analysis. Ethical guidelines were followed at all stages of the study.

Results: Callers both enhanced and hindered interactions. Nurses' professional skills, such as communication skills, nurse-led control over the call and the nurses' capabilities, enabled positive interactions. Disturbing background sounds, communication problems and service system failures made the telephone interactions challenging. Achieving connection with callers, callers who had supportive family members and a supportive organisational structure were features of successful interactions.

Study limitations: As all nine participants were recruited from one call centre, the findings are not directly transferable to another environment.

Conclusions: The results reveal that nurse-caller interactions are affected by several issues concerning the callers and the nurses' skills. Communication problems were often present when telenurses were unable to provide the services callers expected due to lacking health and medical care resources. Family members could be considered important participants in telephone communication with nurses, though further research should examine the possible benefits of interacting with family members.

Practical implications: Based on the results of this study, telenurses could benefit from training that focuses on the communication skills that are needed for telephone nursing and the tools needed to meet individual callers' needs. Work environments could also better support caller-nurse interactions. Organisations should provide more resources for telephone nursing in order to promote positive interactions.

Keywords: Telephone nursing; telenursing; telenurse; caller; interaction; experience 


\section{INTRODUCTION}

Telenursing, also known as telephone nursing, has expanded over the past decade and is projected to expand in the future [1, 2], but little is known about the telenurses' interactions with callers or how nurses compensate for the absence of visual contact during consultations. Telenursing is a modern form of nursing that offers nurses the opportunity to work independently and to develop new skills [3]. Telenursing services are easily accessible by everyone. Telenurses give personal advice, promote self-care, triage health problems and refer callers to the appropriate level of health care [4]. Additionally, telenurses must have a wide range of nursing and medical knowledge and are expected to be sensitive and composed [5].

Telenursing is provided by nurses who work in primary health care, hospitals and emergency departments and in call centres. Some of the nurses' calls are pre-planned; for example, telephone interventions after bypass surgery [6]. However, most calls are unplanned, often initiated by patients, and cover a vast range of health issues. In Finland, if a person requires medical assistance, they may call public primary-care centres during working hours, public emergency hospitals after hours or private clinics at any time. Patient education has become more demanding due to shorter treatment times, a proliferation of clinical treatments, increased patient knowledge and an increase in information technologies [7].

Telenurses spend long periods of time in front of computers with headsets. They have reported feeling stressed and have described their work as monotonous [8]. Previous studies $[9,10,11]$ have indicated that telenurses' working conditions can be stressful, which might threaten patient safety [9]. Stress in telenursing can cause cognitive failures and may hinder nurses' abilities to perform informationprocessing tasks [12]. Lack of feedback limits telenurses' opportunities for professional growth [2], and a need for feedback has been reported $[2,9]$.

Telenursing can be challenging due to the lack of visual contact, making nurses unable to use their senses of sight, touch and smell [13, 9, 3]. Therefore, telenurses need to read between the lines and notice the caller's tone of voice and use of pauses [5]. To manage the lack of visual cues, telenurses have developed alternative interactional skills, such as voice modulating, transposing care over the phone and listening for physical signs and background sounds. Good communication skills are essential for telenursing and help to improve patient safety [9]. 
Communication involves transferring verbal and non-verbal messages between a sender and receiver, but the intended meaning may differ from the imposed meaning [14]. Telenursing is very different from bedside nursing and therefore requires special communication skills [15]. Previous studies have revealed a need to improve telenurses' communication skills $[16,17,18]$. Investigations into malpractice claims in telephone nursing have indicated that the most common reasons for complaint involve the telenurses' failure to listen to the caller, failure to communicate relevant issues concerning the caller's health problem and failure to ask sufficient questions [16].

Along with the nurses' communication skills, the callers' qualities, including their physical and emotional state, might affect the interaction between nurse and caller [19]. Gender can also influence communication; it has been reported that, interactions between nurses and callers are easier and smoother when both are female, while male callers have showed disrespect or have been reluctant to seek care from telenurses [20].

Many studies have addressed telenurses' work experiences, but few have examined how these experiences might affect interactions with callers. The aim of the study was to describe telenurses' experiences of their work. In this paper we report results from the research question: how telenurses experience interaction with callers.

In this study, the person who makes the phone call is referred to as 'the caller' and can be either the patient or a family member who calls on behalf of their relative.

\section{METHODS}

A qualitative descriptive design was chosen for this study as it is an effective method of investigating people's thoughts and experiences. The focus of qualitive study is on developing an understanding of individuals' experiences in their own context; generalising the findings for a wider population is not the goal [21].

\subsection{Participants}

Nine of the 13 registered nurses who work in one call centre that handles calls on behalf of the primary-care health centres and district hospital in a mid-sized town in Finland participated in the study. Participants were all female, aged 26 - 57 years, with an average age of 50 years. The average total nursing experience was 21 years, with individual nurses having between 2.5 - 33 years of experience. Average experience in telenursing was 4.5 years, with individual nurses having between 
8 months -10 years of experience. At the time this study was completed, the nurses' work consisted solely of telenursing. Participants also had access to medical databases to help guide their decisionmaking.

\subsection{Procedure}

The organisation of the call centre was asked a permission for the research. The first author held an information meeting to the nurses at the call centre to gather participants for the study. The nurses who were absent were invited to participate by their supervisor. Nine of the nurses were willing to participate in the study, hence four nurses refrained from participating. Data collection was carried out in 2017 through audio-recorded open telephone interviews. Individual interview technique was chosen to achieve the best possible connection with each participant [22]. Telephone interviews were conducted by the first author. All participants were asked to speak freely about their experiences of telenursing. The first author consulted an interview guide with a list of open-ended questions, each based on literature and professional expertise, and provided helpful questions when required (see Table 1) [23, 22]. The questions had been pilot tested with the group of nurses. Interviews lasted between 40 and 90 minutes, were transcribed verbatim and were coded to ensure anonymity. During the interviews, the first author made field notes to support the analysis.

The resulting data was analysed through inductive content analysis [24]. The text was read through several times to ensure the data was properly understood. Following this process, the researchers identified 1,751 condensed units of meaning to answer the research question (Table 2 shows an example of this analysis). The meaning units were then evaluated and sorted into 12 subcategories and, finally, into four categories (see Figure 1). Throughout this process, the transcribed text was repeatedly consulted to ensure the participants' original meaning was maintained. Analysis was completed manually in several stages to avoid fatigue and error [21, 22, 24]. The analysis was conducted by the first author and regularly shared with the research group.

\subsection{Ethics}

Organisation granted permission for the research. Participation was voluntary. Participants gave their informed consent based on the information provided both in writing and orally. Participants had the right to withdraw from the study at any time. According to the Finnish Advisory Board on Research Integrity guidelines, an ethical review was not required as the study did not involve patients [25]. 


\section{RESULTS}

\subsection{Contradictory Impact of the Caller}

Caller-related hindrances and enhancements, and callers' individual features, produced contradictory aspects of the nurse-caller interaction.

Caller-related hindrances appeared when a caller expressed a negative attitude or behaved inappropriately. Callers' inappropriate behaviours seemed easier for callers to display via telephone than via face-to-face communication and negatively affected the nurses' behaviour and spirits. One nurse stated, 'Although you try to create a peaceful and good interaction, if the caller is so agitated that he has decided the situation will go how he wants, and if this is impossible to arrange, the interaction leads to nothing' (N1). Callers decelerated the interaction, for example, by stepping away from the phone to search for an identity card. Sharing insufficient information and expressing difficult emotions, like anger, made the interaction challenging. Callers engaged in driving or smoking during the call also made the interaction more difficult. Callers were asked to park their cars or use a handsfree connection. Disconnecting the call was a caller's way of ending an unpleasant interaction.

Caller-related enhancement occurred when the caller immediately provided a reason for the call and communicated smoothly with the nurse. Callers' self-tests (fever, blood sugar) and assessments (palpating the stomach, checking skin colour) provided more information and facilitated the interaction. Minimal background noise improved the interaction and callers often sought peaceful places for calling. However, some background noises gave nurses more information about the caller's location, condition and possible companions. For example, 'When you hear a child wheezing in the background and coughing horribly, of course they need to be advised to go to the emergency immediately. The sounds help the assessment' (N7).

A caller's individual features, such as age, sex, physical symptoms and tone of voice, influenced the interaction. The caller's tone of voice reflected emotions like fear and hastiness: 'If the caller is lachrymose or especially anxious, the tone of voice [...] reflects the caller's situation' (N6). Nurses had to consider each caller's abilities and whether that person lived alone or was far from a hospital. Some features, like a caller's impaired hearing, prohibited interaction. Men were described as a challenging group of callers due to their tendency to underrate symptoms and their general reluctance to seek care. Interaction with each caller was unique, with call duration depending on caller and often lasting longer with older callers.

\subsection{Nurses' Professional Skills Enabling Interaction}


Nurses' communication skills, nurse-led control over the call and nurses' capabilities enabled good interaction via telephone.

Nurses' communication skills, including their ability to listen and use intuition and sensitivity to understand the caller's situation without the benefit of eye contact, improved telephone interactions. Nurses had to visualise the callers in their minds to bypass any communication barriers. Nurses with more work experience described the advantages of earlier clinical experiences in visualising the callers' symptoms: 'I get images based on my all-around clinical work experience, so I can somehow see the caller through the eyes of my soul' (N7). By asking the right questions, nurses kept the focus on essential issues to improve the interaction. Friendliness, empathy, politeness and openness were listed as important qualities to display. Giving each caller enough time to explain the situation at the beginning of the call, being genuinely present, using humour when appropriate and greeting the caller according to the time of day were nurses' methods of promoting positive interactions. One nurse explained that 'creating a good atmosphere is important in telenursing, so that you are not like a robot, but a genuine person answering the calls' (N8).

In difficult situations, a nurse's ability to discuss, conciliate and control her own behaviour enabled and promoted the interaction. Remaining calm in every situation was an important part of successfully finishing each call. The nurses noted callers' emotions and respected each caller's free will. The nurses wanted to communicate in suitable ways with each caller, which meant using appropriate words depending on the caller's age, accent or level of education. Each nurse utilised her own voice as a communication tool by adjusting her rhythm and tone to match the situation; for example, to calm the caller or emphasise given orders. As one nurse explained, 'If the situation concerns me - for example, an urgent emergency case where I am worried about whether the caller understands my orders, that there is a need to go to the emergency immediately - then I try to speak slowly, emphasise words... like articulating clearly' (N8). Based solely on verbal communication, nurses assessed caller's condition and urgency to identify whether the caller overstated or understated their symptoms. Gathering the caller's main concern from the conversation and sharing detailed information were important parts of interaction.

Nurse-led control over the call was needed to keep interactions rational. When callers spoke too much or talked about irrelevant issues, the nurses had to interrupt. One nurse said, 'If the caller's story is too long and broad, so that I can't find the common thread, I break into the speech and ask a detailed question' (N2). When a caller became too agitated to communicate clearly, the nurses found it helpful to put the caller on hold, giving both parties time to calm down before continuing the interaction. Disconnecting a call was a nurse's way of ending redundant conversations or 
unsuccessful interactions. Nurses were responsible for leading each interaction and each nurse's professional skills determined the direction of the interaction and increased the nurse's credibility. If a caller recognised one nurse's voice, that nurse was responsible for suggesting the caller speak with another nurse. Nurses had a duty to appraise each caller's overall situation and verify whether the caller correctly understood the situation.

Nurses' capabilities. Interactions evoked different emotions in the nurses, including concern, sadness, discomfort and delight. Emotions and concerns within a nurse's personal life created challenging interactions, although the nurses tried to remain neutral. Concentrating on interactions was exhausting for the nurses, and resting between calls helped them to continue working, especially after difficult calls. The nurses wanted to reflect on failed interactions to understand the reasons for each failure. Good workplace ergonomics; the ability to move and stretch during telephone interactions, helped the nurses to better concentrate on their callers. Interaction without visual contact gave the nurses freedom to express their emotions through body language. One nurse explained, 'Sometimes there are situations when a caller is irritating and I almost rip my hair off, but I still handle the call. I have my ways of hiding my feelings so the caller will not hear my irritation' (N7).

\subsection{Challenging Interactions}

Disturbing background sounds, communication problems and service system shortcomings challenged the telephone interactions.

Disturbing background sounds caused by radio, TV, children, other people and colleagues made it difficult for nurses to hear their callers or to be certain whether the caller was listening or understanding to what the nurse was saying. As one nurse mentioned, 'Of course, if a baby cries while on her mom's lap, it only disturbs the call' (N7). Nurses sometimes had to request the caller remove the background noise or look for a quieter place. Callers calling from their workplaces or while shopping, created unnecessary background noise. When the background sounds were loud, the nurses had to speak more clearly and use stronger intonation. Insufficient soundproofing at the nurses' workplace occasionally made it possible for callers to hear the other nurses in the background, which could lead to a breach of confidentiality. Even the introduction of new, close-fitting earphones did not muffle the background noise and the nurses described difficulties concentrating on their interactions.

Communication problems. Lack of a common language and eye contact restricted interactions. The nurses found it difficult to assess a caller's actual condition, especially skin-related problems, based only on the information provided orally. Mutual understanding was challenging, and it was not always 
possible to complete every interaction. The rhythm of discourse between nurse and caller could also be complicated; for example, 'Some callers speak over my voice all the time and then I do not manage to say what I want' (N1). Inadequate communication, caused by a caller not being able to describe their symptoms or a nurse failing to ask the right questions, could risk a patient's safety. Communicating through family members was sometimes challenging and confusing, if family member and person in need provided different information. 'Often, when the first person has said something and then the other one comes to the phone and I ask, "could you tell me about the issue in your own words?", they talk about something different. Then it is difficult to assess the situation over the phone' (N1). The nurses also described situations in which a family member called and the person in need took part in the conversation by shouting and commenting in the background. Confidentiality requirements made communicating via family members challenging because the nurses were unable to provide certain details to the family member. Sometimes a spouse, usually the wife, became enraged when the nurse asked if the caller had the patient's permission to call.

Service system shortcomings, including a lack of available physicians' appointments, technical problems and long queues, all complicated the nurses' interactions with callers. Explaining the lack of available appointments took time away from the conversation and callers became irritated with the nurses. Technical problems, a bad telephone connection or slow computer programs, prevented interactions from being completed and callers were required to wait or call back later. The queues negatively influenced interactions and callers sometimes gave negative feedback or shouted at the nurses. Nurses tried to communicate more quickly, knowing the callers had waited in the queue for a long time, but they also wanted to communicate without rushing and tried to ignore the queues by hiding the queue line on their computers. When queues were long, nurses often had to begin their conversations by calming the callers. As one nurse explained, 'The caller had to wait too long before he got to speak to the nurse, which is why his emotions were raised. Then I had to consider how I could calm him down as soon as possible' (N3).

\subsection{Successful Interactions}

Achieving a connection with callers, callers who had supportive family members and supportive organisational structures were features of successful interactions.

Achieving connection. The nurses felt successful when interactions went smoothly; when they asked the right questions, spoke in a suitable tone of voice and properly listened to the caller; and when both parties were happy at the end of the call. Nurses also felt happy when callers were thankful and satisfied. One nurse explained that 'it feels nice when a caller says, "oh, this was such a good call, I 
already feel so much better". Then I feel I have succeeded in the interaction' (N3). Experiencing a positive interaction could increase a caller's level of satisfaction, and simply talking to someone helped callers even when no solution was found. The nurses tried to create an atmosphere where callers felt heard and where their concerns were taken seriously. Even when the interaction was challenging, or the case otherwise difficult, the nurses continued to help their callers.

Supportive family members. Callers reaching out on behalf of their relatives occurred frequently. Family members often waited on the line and, when the call was answered, the person in need took over or they would both continue the call. Additional information gathered from family members was sometimes life-saving. If the person in need was not able to speak because of pain, the nurse gathered information from a family member. Family members often called when the person in need could not hear well or suffered a decline in memory. In addition, some men found it difficult to interact via telephone and wanted their wives to speak for them. Nurses also contacted family members if they felt the person in need did not understand the instructions. Family members' attendance was helpful in assessing a patient's condition. As one nurse illustrated, 'A person had fallen and there was a cut on their head, but of course he could not see it by himself. There was a family member who looked at it and told me what kind of cut it was' (N1). However, the nurses always asked to speak with the person in need, where appropriate.

Supportive organisational structures. From the caller's medical records, nurses were able to access information from earlier calls, easing the interaction. 'The drug-abuser can immediately call again, but when I have already written the situation in the medical records, my colleague sees it and can repeat the same instructions' (N6). Having access to physicians' orders also helped the nurses explain the situation to a caller. Calls sometimes changed direction when the nurse opened the patient's medical records and realised the caller had not shared all relevant details. The nurses' ability to cooperate with social services and the public safety call centre supported the interaction when there were concerns regarding the caller's medical condition. Being able to ask emergency responders to go to a caller's home reduced the pressure on nurses to solve the caller's issue via telephone interaction only. Common directives within the work environment also helped improve interaction as nurses followed the same guidelines with their callers.

\section{STRENGHTS AND LIMITATIONS}

Interviews were comprehensive and provided rich data about the telenurses' experiences. However, as there were only nine participants, all of whom had been recruited from one call centre, the findings 
are not directly transferable to another environment [24]. Transferability has been increased by reporting information regarding the context, participants, methods of data collection and analysis [24]. In choosing this call centre, the authors' goal was to avoid any conflict of interests, as the first author had previous experience in telenursing. The participants were not familiar to the interviewer. While this previous experience could be considered a limitation, it can also be seen as a strength as the participating nurses spoke frankly in their interviews, enriching the credibility of the study's data.

All participants were female, which could be considered a limitation of the study's credibility [24]. However, in Finland, the vast majority of nursing graduates are women and, as all of the nurses who worked for the study organisation were female, the participants were representative of the population. It should also be noted that the findings reflect only the perspectives of the telenurses; callers were not included in this study.

\section{DISCUSSION AND CONCLUSION}

\subsection{Discussion}

While this study builds upon the existing knowledge of telenursing, it also provides new information from the telenurses' perspective regarding nurse-caller interactions. The results indicate that nursecaller interactions are affected by several issues, including the caller's personality, the nurse's professional skills, the caller's family members and the telenursing organisational structure. Interactions are prone to disturbances, yet there are positive aspects.

One issue identified by this study is the contradictory impact of the caller. While the caller is the key focus of each call, in accordance with earlier findings, this study identifies how callers' inappropriate behaviours complicate the interactions [5]. Verbaly abusive callers add to nurses' emotional workload [26] and threaten the callers' own safety [9]. This and previous studies [15] show that a caller's ability to promote their cause can support the interaction, such as performing self-tests to gather information; however, callers may also hinder the interaction through their actions, such as driving or smoking during the call. The need to call can occur anywhere, but the nurses who participated in this study felt it was their responsibility to advise callers to end the call and contact again once the caller was in appropriate location. As callers can risk their own and other people's safety while driving, advising callers is a matter of traffic safety. This example highlights the special components of telephone interactions. 
In this study, nurses' professional skills had a positive impact on the interactions. One key element was the nurses' ability to extract relevant details from irrelevant information [27], which could be hampered by a lack of shared language and eye contact with the patient $[13,9,3,10]$. Based on the telenurses' interactions with callers, this study supports previous findings and indicates that nurses visualise callers $[9,15,27]$ and their symptoms [27, 28], which is a key component of telenursing and is mostly related to the nurses' clinical work experience [27, 28]. Listening to background sounds $[29,15]$, the caller's breathing and tone of voice [5], helped the nurses to visualise symptoms and gather information [15], demonstrating the richness of telenursing and telephone interaction.

This and previous studies [10, 29] indicate that remaining calm is an important skill within professional nursing, and callers expect the nurses to remain calm and friendly at all times [4]. However, staying calm and friendly can be challenging when callers display inappropriate behaviours. This study demonstrates how the nurses lead calls through a balance of listening and controlling the callers' speech. As an extreme method, telenurses handle difficult callers by placing calls on hold when inappropriate behaviour is displayed, a novel approach that shows the nurses' rectitude. In this study, disconnecting a call is a method of ending redundant conversations, for both the nurses and their callers, showing the frailty of telephone interaction. An antonym of ending the call, humour was used as a tool of interaction in this study.

The findings of this study suggest that interactions can fail due to communication problems. One possible solution to equalise interactions is the implementation of a decision support tool, used in Sweden and the UK. The tool can ease the telenurses' workload by guiding the nurses' decisionmaking process and may also have an impact on the interaction. Research has shown that these programmes can support nurses when they are stressed, tired or subject to call repetition [30]. However, the challenges of interacting with family members, as shown in this study, are more complicated. Instances of family members providing ambivalent information or disturbing the interaction by commenting in the background are specific situations experienced within telenursing that should be addressed through education. Telenurses would benefit from training that focuses on questioning, listening and counselling skills [15].

Although telephone interactions were shown to be challenging, this study also demonstrates that there are benefits to telenursing. Being invisible to the callers provides nurses with the option to rest between calls and to use body language to express their emotions, both of which can promote positive interactions. Previous studies have indicated that invisibility can protect a nurse's professional image, especially during particularly emotional calls [26]. The findings of this study suggest that the nurses' 
work environment, including workplace ergonomics and the availability of space to rest, can support both work hygiene and the nurses' interactions with callers. Considering these results, in conjunction with the stressful and monotonous nature of telenursing, more attention should be directed toward planning the nurses' work environment in order to support nurse-caller interactions.

The ability to listen to background sounds is an important skill in telenursing $[2,15]$, though this study indicates that background sounds can be both useful and disturbing. Insufficient soundproofing of the work environment can detract from an interaction, which is useful information for planning telenursing environments. The callers' disturbing background sounds are not something nurses can control, but adequate soundproofing of the work environment would help control the sounds in the nurses' background. Lack of resources, such as available physicians' appointments, and long queues can evoke frustration in both callers and nurses, which has been suggested previously [31, 10, 32]. This study highlights the impact of these shortcomings on nurse-caller interactions, findings that cannot be overstressed. Based on these results, it would be reasonable to state that telenurses do not have a solid base upon which to create a positive atmosphere for interaction, as the callers are required to queue, and appointments are unavailable.

Holistic nursing [27] and successful interaction [5, 26] are sources of job satisfaction in telephone nursing. In this study, immediate positive feedback from callers was listed as a component of successful interaction. Knowing that they are helping their callers encourages the nurses to continue working, even in challenging situations. Considering these results, regularly collecting customer feedback might be one method of supporting nurses in their interactions with callers.

This study highlights family members as important informants who can carry out activities during the call and can help support the interaction. This and previous studies have also addressed some of the negative aspects of interacting through a third party, such as communication difficulties $[9,10]$ and ethical concerns [33]. However, this study shows that, in urgent situations, family members are more likely to place the call, which can be life-saving. Therefore, it is important to further evaluate nursefamily member interactions in telenursing and the possible benefits of such interactions. This study indicates that women tend to call on behalf of their husbands, supporting the idea that female callers take responsibility for their family members' health [34]. Telenurses are generally female and previous studies have indicated that their methods of communicating are more appealing to female callers [20, 35], raising the question of male callers' position in telenursing.

\subsection{Conclusion}


The present study indicates that nurses' communication skills, clinical work experience and willingness to help all facilitate successful interactions. Background sounds, insufficient workplace soundproofing and communication problems may detract from an interaction. Some challenges of nurse-caller interactions may be caused by service system inadequacies. There is a need for further research into telenurses' interactions with family members, as they are important informants despite the challenges they present.

\subsection{Practical Implications}

To enable high-quality interaction, telephone nurses would benefit from training that focuses on the effective use of voice, expressing care over the phone and communication skills in the absence of eye contact. Nurses require specific tools for each individual encounter with a caller, including family members and challenging personalities. The work environment and facilities should support nursecaller interactions by providing opportunities to rest between calls and avoid disturbing noises.

Funding: This research did not receive any specific grant from funding agencies in the public, commercial or not-for-profit sectors.

Declarations of interest: none.

\section{Acknowledgments}

We would like to thank the nurses and their supervisor who willingly participated in this study and shared their experiences.

\section{Ethical issues}

We confirm all patient/personal identifiers have been removed or disguised so the patient/persons described are not identifiable and cannot be identified through the details of the story. 


\section{REFERENCES}

1. Johnson S, Wilhelmsson S, Börjeson S, Lindberg M. Improvement of communication and interpersonal competence in telenursing - development of self-assessment tool. J Clin Nurs $2015 ; 24: 1489-1501$.

2. Greenberg M.E. A comprehensive model of the process of telephone nursing. J Adv Nurs 2009; 65:2621-29.

3. Snooks H.A, Williams A.M, Griffiths L.J, Peconi J, Rance J, Snelgrove S, Sarangi S, Wainwright P, Cheung W. Real nursing? The development of telenursing, J Adv Nurs 2008; 61:631-40.

4. Ström M, Marklund B, Hildingh C. Caller's perceptions of receiving advice via a medical help care line. Scand J Caring Sci 2009; 23:682-90.

5. Holmström I, Dall'Alba G. 'Carer and gatekeeper' -- conflicting demands in nurses' experiences of telephone advisory services. Scand J Caring Sci 2002; 16:142-48.

6. Hartford K. Telenursing and patients' recovery from bypass surgery. J Adv Nurs 2005; 50: 459- 468.

7. Eloranta S, Leino-Kilpi H, Katajisto J. Does the empowerment patient education realize from the perspective of the nurses. Hoitotiede 2014; 26:63-73. [in Finnish]

8. Knowles E, O’Cathain A, Morrell J, Munro J, Nicholl JP. NHS Direct and nurses opportunity or monotony? Int J Nurs Stud 2002; 39:857- 866.

9. Röing M, Rosenqvist U, Holmström I.K. Threats to patient safety in telenursing as revealed in Swedish telenurses' reflections on their dialogues. Scand J Caring Sci 2013; 27:969-76.

10. Wahlberg A.C, Cedersund E, Wredling R. Telephone nurses' experiences of problems with telephone advice in Sweden. J Clin Nurs 2003; 12:37-45. 
11. Reinhardt A.C. The impact of work environment on telephone advice nursing. Clin Nurs Res 2010; 19:289-310.

12. Allan J.L, Farquharson B, Johnston D.W, Jones M.C, Choudhary C.J, Johnston M. Stress in telephone helpline nurses is associated with failures of concentration, attention and memory, and with more conservative referral decisions. Br J Psychol 2014; 105:200-13.

13. Ruston A. Interpreting and managing risk in a machine bureaucracy: professional decision making in NHS Direct. Health Risk Soc 2006; 8:257-71.

14. Fielding R. Clinical communication skills. Hong Kong University Press, Hong Kong,1995.

15. Pettinari C.J, Jessopp L. 'Your ears become your eyes': managing the absence of visibility in NHS Direct. J Adv Nurs 2001; 36:668-75.

16. Ernesäter A, Winblad U, Engström M, Holmström I.K. Malpractice claims regarding calls to Swedish telephone advice nursing: what went wrong and why? J Telemed Telecare 2012; 18:379-83.

17. Moscato S.R, Valanis B, Gullion C.M, Tanner C, Shapiro S.E, Izumi S. Predictors of patient satisfaction with telephone nursing services. Clin Nurs Res 2007; 16:119-37.

18. Valanis B.G, Gullion C.M, Moscato S.R, Tanner C, Izumi S, Shapiro S.E. Predicting patient follow-through on telephone nursing advice, Clin Nurs Res 2007; 16:251-69.

19. Leprohon J, Patel V.L. Decision-making strategies for telephone triage in emergency medical services. Med Decis Making 1995; 15:240-253.

20. Höglund A, Holmström I. 'It's easier to talk to a woman', Aspects of gender in Swedish telenursing. J Clin Nurs 2008; 17:2979-86.

21. Fick U. An introduction to qualitative research. 2014, Fifth edition, Sage, London.

22. Polit D, Beck C. Nursing Research, Generating and Assessing Evidence for Nursing Practice. 2012, Ninth Edition, Lippincott Williams \& Wilkins, China. 
23. Parahoo K. Nursing Research, Principles, process and issues. 2014, Third edition, Palgrave Macmillan, China.

24. Graneheim U.H, Lundman B. Qualitative content analysis in nursing research: concepts, procedures and measures to achieve trustworthiness. Nurse Educ Today 2004; 24:105-112.

25. TENK 2012. https://www.tenk.fi/sites/tenk.fi/files/HTK ohje 2012.pdf

26. Weir H, Waddington K. Continuities in caring? Emotion work in a NHS Direct call centre. Nurs Inq 2007; 15:67-77.

27. Snelgrove SR. Nursing work in NHS Direct: constructing a nursing identity in the callcentre environment. Nurs Inq 2009; 16:355-65.

28. Edwards B. Seeing is believing — picture building: a key component of telephone triage. J Clin Nurs 1998; 7:51-57.

29. Kaminsky E, Rosenqvist U, Holmström I. Telenurses' understanding of work: detective or educator? J Adv Nurs 2009; 65:382-90.

30. Ernesäter A, Holmström I, Engström M. Telenurses` experiences of working with computerized decision support: Supporting, inhibiting, and quality improving. J Adv Nurs 2009; 65:1074-1083.

31. Länneström L, Wallman T, Söderbäck M. Nurses' experiences of managing sick-listing issues in telephone advisory services at primary health care centres. Scand J Caring 2013; 27:857-63.

32. Valanis B, Moscato S.R, Tanner C, Shapiro S, Izumi S, David M, Mayo A. Making it work: Organization and processes of telephone nursing advice services. J Nurs Adm 2003; $33: 216-23$.

33. Holmström I, Höglund A.T. The faceless encounter: ethical dilemmas in telephone nursing. J Clin Nurs 2007; 16:1865-71.

34. Kaminsky E, Carlsson M, Höglund A.T, Holmström I. Paediatric health calls to Swedish telenurses: A descriptive study of content and outcome. J Telemed Telecare 2010; 16:454-7. 
35. Hakimnia R, Holmström I, Carlsson M, Höglund A. Exploring the communication between telenurse and caller - A critical discourse analysis. Int J Qual Stud Health well-being 2014; 9:24255.

Table 1. Interview guide

\begin{tabular}{|c|c|}
\hline Interview question & "Tell me about your work as a telenurse" \\
\hline Additional questions & $\begin{array}{l}\text { Can you tell me about: } \\
\text { - the stages of the call? } \\
\text { - the interaction in telenursing? } \\
\text { - the skills needed in telenursing? } \\
\text { - the professional knowledge and development in telenursing? } \\
\text { - the organization, resources and working environment? }\end{array}$ \\
\hline
\end{tabular}

Table 2. Example of analysis.

\begin{tabular}{|c|c|c|c|}
\hline Examples of meaning units & $\begin{array}{l}\text { Examples of condensed } \\
\text { meaning units }\end{array}$ & $\begin{array}{l}\text { Examples of } \\
\text { subcategories }\end{array}$ & $\begin{array}{l}\text { Main } \\
\text { category }\end{array}$ \\
\hline \multirow{2}{*}{$\begin{array}{l}\text { 'Some callers have a strong, } \\
\text { irascible attitude when they call. } \\
\text { They know what they want, and } \\
\text { they want to get it - for example, } \\
\text { an appointment. But if there are } \\
\text { none available, that is a problem.' }\end{array}$} & $\begin{array}{l}\text { Some callers have an } \\
\text { irascible attitude about } \\
\text { getting what they want. }\end{array}$ & \multirow[t]{5}{*}{$\begin{array}{l}\text { Caller-related } \\
\text { hindrances }\end{array}$} & \multirow[t]{9}{*}{$\begin{array}{l}\text { Contradictory } \\
\text { impact of the } \\
\text { caller }\end{array}$} \\
\hline & $\begin{array}{l}\text { It is problematic when a } \\
\text { caller cannot get what s/he } \\
\text { wants. }\end{array}$ & & \\
\hline \multirow{3}{*}{$\begin{array}{l}\text { 'Of course, some callers [...] do } \\
\text { not want to tell me their name, } \\
\text { saying, "I really don't want to tell } \\
\text { you my name". They do not tell } \\
\text { me enough so I can help with their } \\
\text { issues and they might immediately } \\
\text { give negative feedback.' }\end{array}$} & $\begin{array}{l}\text { Sometimes a caller does not } \\
\text { want to share his/her name. }\end{array}$ & & \\
\hline & $\begin{array}{l}\text { Sometimes a caller does not } \\
\text { give enough information for } \\
\text { the nurse to properly assist. }\end{array}$ & & \\
\hline & $\begin{array}{l}\text { Feedback from callers can } \\
\text { immediately be negative. }\end{array}$ & & \\
\hline \multirow{2}{*}{$\begin{array}{l}\text { 'Interaction is fluent, and things } \\
\text { get on well when the caller can tell } \\
\text { me her/his story clearly and } \\
\text { understands what I say.' }\end{array}$} & $\begin{array}{l}\text { Things get on well when a } \\
\text { caller tells their story clearly. }\end{array}$ & \multirow[t]{2}{*}{$\begin{array}{l}\text { Caller-related } \\
\text { enhancements }\end{array}$} & \\
\hline & $\begin{array}{l}\text { Things get on well when a } \\
\text { caller understands what the } \\
\text { nurse says. }\end{array}$ & & \\
\hline $\begin{array}{l}\text { 'When the caller is older, or the } \\
\text { parlance is unclear - I speak as } \\
\text { clearly as possible.' }\end{array}$ & $\begin{array}{l}\text { The nurse speaks clearly if } \\
\text { the caller is an older person. }\end{array}$ & \multirow[t]{2}{*}{$\begin{array}{l}\text { Caller's } \\
\text { individual } \\
\text { features }\end{array}$} & \\
\hline $\begin{array}{l}\text { 'Generally, the older men }[\ldots] \\
\text { understate their symptoms.' }\end{array}$ & $\begin{array}{l}\text { Older men often understate } \\
\text { their symptoms. }\end{array}$ & & \\
\hline
\end{tabular}




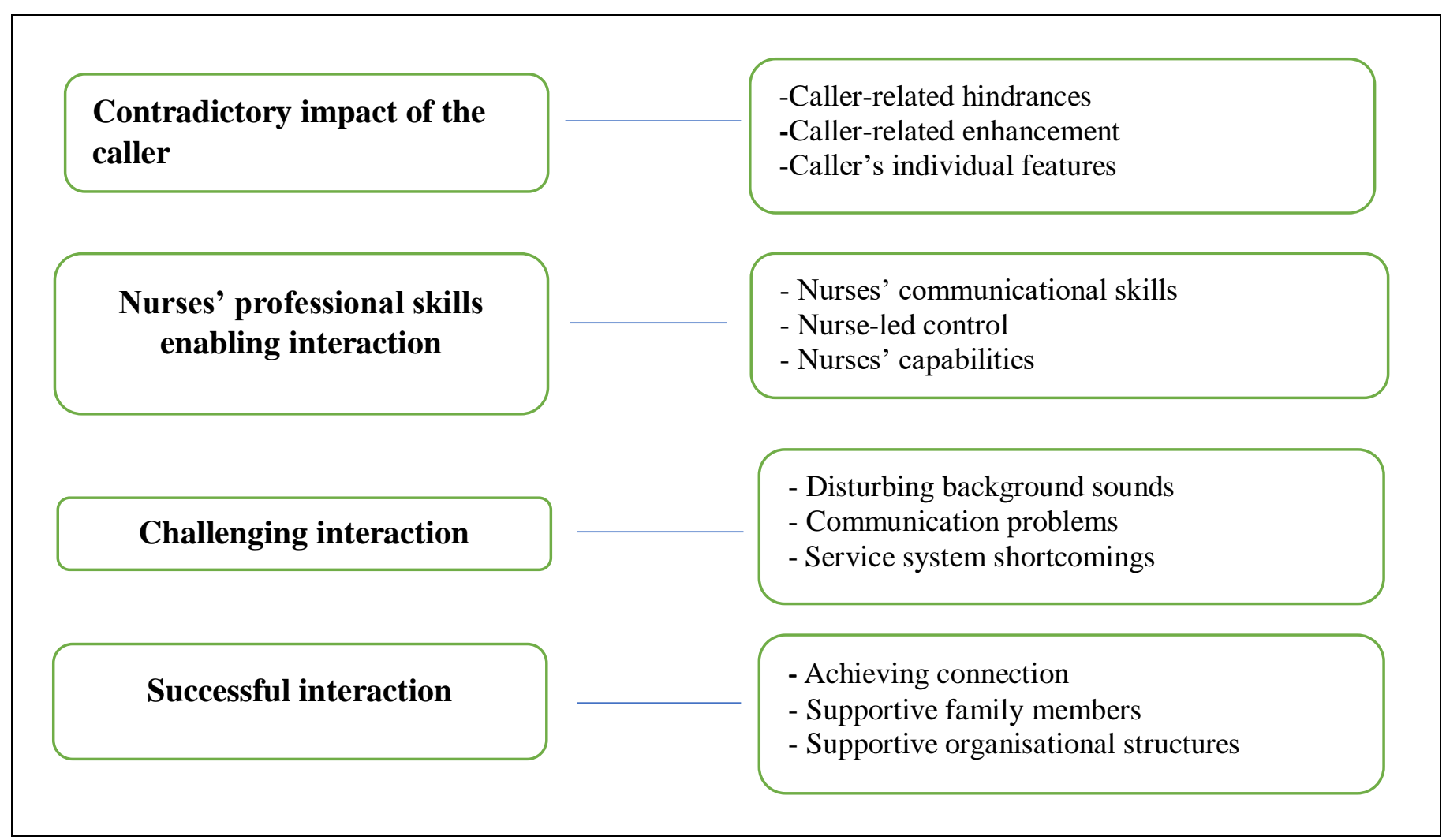

Figure 1. Telephone nurses' experiences of the interaction with callers (categories, subcategories) 